\title{
Refractive index of InGaN/GaN quantum well
}

\author{
Mandy M. Y. Leung, Aleksandra B. Djurisić, and E. Herbert Lia) \\ Department of Electrical \& Electronic Engineering, University of Hong Kong, Pokfulam Road, Hong Kong
}

(Received 22 June 1998; accepted for publication 2 September 1998)

\begin{abstract}
In this article, the optical properties of the $\operatorname{In}_{x} \mathrm{Ga}_{1-x} \mathrm{~N} / \mathrm{GaN}$ quantum well $(\mathrm{QW})$ are investigated. The refractive index spectrum of a $\mathrm{QW}$ is essential to the design and implementation of optoelectronic devices. Yet, the refractive index of the InGaN/GaN QW system over a wide spectral range has been unavailable so far. This article presents a comprehensive model, which includes the exciton effect and most of the major critical points, to calculate the complex index of refraction of the InGaN/GaN QW at room temperature. The calculations have been performed for QW's with various alloy compositions and well widths in the spectral range from 1 to $9 \mathrm{eV}$. The model presented here fully considers transitions near the band edge and above barrier gap contributions. (C) 1998 American Institute of Physics. [S0021-8979(98)06123-4]
\end{abstract}

\section{INTRODUCTION}

As a result of the growing interest in blue light emitting diodes and laser diodes for use in full color displays and high packing density data storage, III-V nitride based materials have become an important topic of study. GaN and related materials, such as the alloy AlGaInN, are III-V nitride semiconductors suitable for light emitting within the range from 0.2 to $0.636 \mu \mathrm{m}$. GaN has a direct band gap of $3.4 \mathrm{eV}$, and the AlGaN and InGaN alloys demonstrate a band gap ranging from 1.95 to $6.2 \mathrm{eV}$, depending on the alloy composition. ${ }^{1}$ Up until now, however, very little has been known about the optical properties of the InGaN/GaN quantum well $(\mathrm{QW})$ system. Since a precise understanding of the optical properties of these materials is essential to effective device operation and design, it is necessary to develop a comprehensive model which deals with the theoretical calculation of the refractive index.

In this work, the effects of mole fraction $(x)$ of indium and the width of the well layer on the refractive index of the $\mathrm{In}_{x} \mathrm{Ga}_{1-x} \mathrm{~N} / \mathrm{GaN}$ square $\mathrm{QW}$ are analyzed. To calculate the refractive index, both the real and imaginary parts of the dielectric function $\epsilon(E)$ as a function of the photon energy $E=\hbar \omega$ over a wide spectral range are used. All transitions, including the excitonic effect, are taken into consideration in describing the QW system in a spectral range between 1 and $9 \mathrm{eV}$. The model used for our theoretical calculation is based on Kramers-Kronig transformation (KKT) and is strongly connected to the band structure of the materials. One special feature of this model is that it takes into account optical responses over a wide range of photon energies. Also, the optical properties described in terms of the complex optical dielectric function are expressed as a function of critical point energies. ${ }^{2}$ Based on the band structure of $\mathrm{In}_{x} \mathrm{Ga}_{1-x} \mathrm{~N} / \mathrm{GaN}$ calculated via the $k \cdot p$ method for wurtzite semiconductors, ${ }^{3}$ only dominant interband transitions are considered. The lowest direct band gap is denoted as $E_{0}$, and the other transitions at energy regions higher than that of $E_{0}$

a)Electronic mail: ehli@eee.hku.hk are denoted as $E_{1 \beta}$ where $\beta=A, B$, and $C$. A full QW calculation is employed for the $E_{0}$ region where the QW effect is the most significant. A continuum state calculation is performed for the higher lying direct transitions in the energy region $E_{1 \beta}$ using the composite interband transition model. ${ }^{4}$

The contributions to the imaginary part of the dielectric function $\epsilon_{2}(E)$ from each of these dominant transitions are considered separately. Various alloy parameters required for calculation have been estimated by the interpolation between $\alpha$-GaN and InN materials due to scarce research results regarding material parameters and dielectric functions of the $\mathrm{In}_{x} \mathrm{Ga}_{1-x} \mathrm{~N}$ alloy. After summing up the contributions to the imaginary part, the real part of dielectric function $\epsilon_{1}(E)$ is obtained by KKT.

This article is organized as follows: the composite model is briefly described in Sec. II where each energy transition is considered separately. In Sec. III, the estimation of parameters is described. This is followed by Sec. IV, where results of the calculated refractive index are presented. Finally, the conclusion is in Sec. V.

\section{THEORETICAL MODEL}

The refractive index $n(E)$ and the extinction coefficient $k(E)$ are the fundamental optical parameters related to the complex dielectric function $\epsilon(E)=\epsilon_{1}(E)+i \epsilon_{2}(E)$, as shown in the following formulas: ${ }^{5}$

$$
\begin{aligned}
& n(E)=\left(\frac{\left[\epsilon_{1}(E)^{2}+\epsilon_{2}(E)^{2}\right]^{1 / 2}+\epsilon_{1}(E)}{2}\right)^{1 / 2}, \\
& k(E)=\left(\frac{\left[\epsilon_{1}(E)^{2}+\epsilon_{2}(E)^{2}\right]^{1 / 2}-\epsilon_{1}(E)}{2}\right)^{1 / 2} .
\end{aligned}
$$

The dielectric function depends on the electronic energyband structure of the semiconductor QW material. Summing up all the contributions, $\epsilon_{2}(E)$ is

$$
\epsilon_{2}(\omega)=\epsilon_{2}^{E_{0}}(\omega)+\epsilon_{2}^{E_{1 \beta}}(\omega),
$$

where $\epsilon_{2}^{E_{0}}$ denotes the contribution to the dielectric function from the low-lying direct gaps $E_{0}$ and $\epsilon_{2}^{E_{1 \beta}}$ denotes the con- 
tribution to the dielectric function from $E_{1 \beta}$ regions. In the following, a theoretical model for the calculation of the refractive index of the III-V nitride semiconductor QW is presented.

\section{A. $E_{0}$ region}

This energy region corresponds to the band-edge region of the QW. Our calculation includes the exciton and the bound-state effects, which are superimposed to the continuum contribution above the barrier gap energy. $\epsilon_{2}^{E_{0}}$ is obtained by summing up all the above contributions, and it can be expressed as follows:

$$
\epsilon_{2}^{E_{0}}(\omega)=\epsilon_{2}^{\text {exc }}(\omega)+\epsilon_{2}^{\text {bound }}(\omega)+\epsilon_{2}^{E_{0} \text { cont }}(\omega),
$$

where $\omega$ is the frequency, $\epsilon_{2}^{\mathrm{exc}}$ is the imaginary part of the dielectric function contributed by the QW $1 S$ exciton derived by the density-matrix approach at the subband edge, $\epsilon_{2}^{\text {bound }}$ is the imaginary part of the dielectric function contributed by the conduction-valence band QW bound states, and $\epsilon_{2}^{E_{0} \text { cont }}$ is the imaginary part of the dielectric function determined by weighting the InGaN well and GaN barrier continuum's at energies above the barrier energy in the multiple QW (MQW) structure without the QW effect.

\section{1. $Q W$ effect}

The QW subband edge states in the $E_{0}$ region can be calculated separately for electrons and holes by an envelope function approximation ${ }^{6}$ using the Ben-Denial and Duke model. ${ }^{7}$ The imaginary part of the dielectric function contributed by the exciton effect $\epsilon_{2}^{\text {exc }}(\omega)$ is based on the $1 S$ bound exciton wave function determined by a perturbativevariational method, ${ }^{8,9}$ using the separate electron and hole bound state at $k_{\|}=0$ as the trial functions.

The imaginary part of the dielectric function contributed by the conduction-valence band bound-state effect without the electron-hole interaction $\epsilon_{2}^{\text {bound }}(\omega)$ is based on the direct interband transition around the absorption edge in the $E_{0}$ region. $\epsilon_{2}^{\text {bound }}(\omega)$ is expressed as

$$
\begin{aligned}
\epsilon_{2}^{\text {bound }}(\omega)= & \frac{e^{2}}{\epsilon_{0} m_{0}^{2} \omega^{2} L_{z}} \sum_{p, q} \int\left|\hat{e} M_{p q}(k)\right|^{2} d k \frac{1}{4 \pi^{2}} \\
& \times \mathscr{L}\left[E_{p}(k)-E_{q}(k)-\hbar \omega\right],
\end{aligned}
$$

where $M_{p q}$ is the interband momentum-matrix element for the wurtzite materials, ${ }^{10} k$ is the transverse wave vector in the direction parallel to the QW layer; $L_{z}$ is the well width of the QW and $\mathscr{B}$ is the Lorentzian broadening factor with half width half maximum $\Gamma_{b}$. The conduction bands are assumed parabolic; for the valence band calculation, we adopt Chuang's model. ${ }^{3}$ However, instead of using the finite-difference scheme stated there, we use Chan's basis expansion method. ${ }^{11}$ A total of 30 band-edge wave functions at $k_{t}=0$ are determined for the expansion in the calculation of the wave function at $k_{t} \neq 0$. The momentum matrix element in Eq. (4) for transverse electric (TE) $(\hat{e}=\hat{x}$ or $\hat{y} \perp c$ axis) and transverse magnetic $(\mathrm{TM})\left(\hat{e}=\hat{z}\right.$ or $\hat{y} \|_{c}$ axis $)$ polarization can be simplified to

$$
\hat{e} M_{p q}(k)=\left\langle\Psi_{p}^{v}\left|\hat{p}_{x}\right| \Psi_{q}^{c}\right\rangle,
$$

and

$$
\hat{e} M_{p q}(k)=\left\langle\Psi_{p}^{v}\left|\hat{p}_{z}\right| \Psi_{q}^{c}\right\rangle,
$$

respectively. For a detailed expression, please refer to Chuang's work. ${ }^{10}$

Derived by the density-matrix approach at the subband edge without the influence of band mixing, $\epsilon_{2}^{\mathrm{exc}}(\omega)$ is expressed as

$$
\begin{aligned}
\epsilon_{2}^{\operatorname{exc}}(\omega)= & \frac{e^{2}}{\epsilon_{0} m_{0}^{2} \omega^{2} L_{z}}\left|\hat{e} M_{11}(k)\right|^{2} \\
& \times\left|\psi_{1 s}(k=0)\right|^{2} \mathscr{C}\left(E_{\mathrm{exc}}-\hbar \omega\right),
\end{aligned}
$$

where $E_{\text {exc }}$ is the exciton transition energy.

\section{Continuum contribution}

The last step in calculating the dielectric function in the $E_{0}$ region is to combine the above contributions with the continuum state contribution. ${ }^{2}$ The lowest direct gap region of the wurtzite type material is assumed to be a critical point of the three-dimensional (3D) $M_{0}$ type. Together with the assumption that the valence and conduction bands are parabolic, the contribution of this low energy gap to the dielectric function, for a single layer (barrier or well), is expressed as

$$
\begin{aligned}
\epsilon^{E_{0} \text { cont }}(\omega)= & \frac{A_{0}}{\left(E_{0}^{\prime}\right)^{3 / 2}} \frac{2-\left(1+\chi_{0}\right)^{1 / 2}-\left(1-\chi_{0}\right)^{1 / 2}}{\chi_{0}^{2}} \\
& +\sum_{n=1}^{\infty} \frac{A_{0}^{\mathrm{ex}}}{n^{3}} \frac{1}{E_{0}^{\prime}-\left(G_{0}^{3 D} / n^{2}\right)-\hbar \omega-i \Gamma_{0}^{\prime}},
\end{aligned}
$$

where

$$
\chi_{0}=\frac{\hbar \omega+i \Gamma_{0}^{\prime}}{E_{0}^{\prime}},
$$

where $\Gamma_{0}^{\prime}$ is the damping energy of the $E_{0}^{\prime}$ critical point, $A_{0}^{\mathrm{ex}}$ is the $3 \mathrm{D}$ exciton strength parameter, and $G_{0}^{3 \mathrm{D}}$ is the $3 \mathrm{D}$ exciton binding energy. The second term of Eq. (8) represents the discrete series of the exciton lines at the $E_{0}$ edge described by Lorentzian line shape. In the employed model, the damping constant $\Gamma_{j}$ (where $j=0,1 A, 1 B$, and $1 C$ ) is replaced by a frequency dependent expression $\Gamma_{j}^{\prime}$ :

$$
\Gamma_{j}^{\prime}(E)=\Gamma_{j} \exp \left[-\alpha_{j}\left(\frac{E-E_{j}}{\Gamma_{j}}\right)^{2}\right] .
$$

It was shown that by using this variable line shape, where the type of broadening is determined by the ratio of parameters $\alpha_{j}$ and $\Gamma_{j}$ (for example, $\alpha_{j} / \Gamma_{j}<0.1$ Lorentzian and $\alpha_{j} / \Gamma_{j}$ $\approx 0.3$ for Gaussian) a better agreement with the experiments can be obtained. ${ }^{12,13}$

In the calculation of the quantum well, the $\epsilon^{E_{0} \text { cont }}(\omega)$ is calculated by weighting the barrier and the well by a ratio of the well width $\left(L_{z}\right)$ to the barrier width $\left(L_{b}\right)$ :

$$
\epsilon^{E_{0} \text { cont }}(\omega)=\frac{1}{L_{z}+L_{b}}\left[L_{z} \epsilon_{(\text {well })}^{E_{0} \text { cont }}(\omega)+L_{b} \epsilon_{(\text {barrier })}^{E_{\text {cont }}}(\omega)\right] \text {. }
$$


Here, $L_{b}$ is taken to be $100 \AA$ for a MQW structure. Thus, contribution of the continuum state transitions to the imaginary part $\epsilon_{2}^{E_{0} \text { cont }}$ of the dielectric function is given by taking the imaginary part of Eq. (11):

$$
\epsilon_{2}^{E_{0} \text { cont }}(\omega)=\operatorname{Im}\left(\epsilon^{E_{0} \text { cont }}\right) .
$$

\section{B. $E_{1 \beta}$ region}

The energy transitions in higher energy regions are denoted as $E_{1 \beta}$. With the assumption that the quantum well effect can be neglected here, only the continuum state contribution is considered in the calculation of the dielectric function. These critical points should be of the 3D $M_{1}$ type. However, as the longitudinal effective mass is much larger than its transverse counterparts, these critical points can be treated as 2D minima $M_{0} .{ }^{2}$ The contribution to $\epsilon(E)$ is expressed as follows:

$$
\begin{aligned}
\epsilon^{E_{1} \mathrm{cont}}= & -\sum_{\beta=A, B, C} B_{1 \beta} \chi_{1 \beta}^{-2} \ln \left(1-\chi_{1 \beta}^{2}\right) \\
& +\sum_{n=1}^{\infty} \frac{B_{1 \beta}^{\mathrm{ex}}}{(2 n-1)^{3}} \frac{1}{E_{1 \beta}-\left[G_{1 \beta}^{2 \mathrm{D}} /(2 n-1)^{2}\right]-E-i \Gamma_{1 \beta}^{\prime}},
\end{aligned}
$$

where

$$
\chi_{1 \beta}=\frac{E+i \Gamma_{1 \beta}^{\prime}}{E_{1 \beta}}
$$

$\Gamma_{1 \beta}^{\prime}$ is the damping given by Eq. (10); $B_{1 \beta}$ are the strength parameters for the $E_{1 \beta}$ transitions, while $B_{1 \beta}^{\mathrm{ex}}$ and $G_{1 \beta}^{2 \mathrm{D}}$ are the strength parameter and the binding energy of the $2 \mathrm{D}$ excitons at the $E_{1 \beta}$ critical points, respectively.

The final $\epsilon^{E_{1} \text { cont }}(\omega)$ is obtained in the same way as the continuum contribution of the $E_{0}$ transition-that is, by weighting the barrier and well regions by a ratio of well width $\left(L_{z}\right)$ to barrier width $\left(L_{b}\right)$. Thus, the imaginary part $\epsilon_{2}^{E_{1} \text { cont }}$ of the continuum contribution to the dielectric function in the $E_{1 \beta}$ region is given by

$$
\epsilon_{2}^{E_{1} \text { cont }}(\omega)=\operatorname{Im}\left(\epsilon^{E_{1} \text { cont }}\right) .
$$

The imaginary part of the total dielectric function $\epsilon_{2}(\omega)$ is obtained by summing up the contributions from the $E_{0}$ and $E_{1 \beta}$ regions given by Eqs. (4), (7), (12), and (15). The real part of the dielectric function is obtained by performing KKT on $\epsilon_{2}(\omega)$.

\section{ESTIMATION OF PARAMETERS}

In the bound state, exciton and continuum state calculations, a set of material parameter values is required. As for the bound state and exciton calculations, this includes parameters such as Luttinger-like valence-band effective mass parameters $A_{i}$ 's and conduction-band effective mass for electrons and static dielectric constants. As there are no data available for the ternary $\operatorname{In}_{x} \mathrm{Ga}_{1-x} \mathrm{~N}$ material parameters, the linear interpolation scheme, i.e., $P\left(\operatorname{In}_{x} \mathrm{Ga}_{1-x} \mathrm{~N}\right)=x P(\operatorname{InN})$ $+(1-x) P(\mathrm{GaN})$, is used for all the parameters except the band gap. The band gap dependence on the alloy composi-
TABLE I. Material parameters for $\alpha-\mathrm{GaN}$ and $\mathrm{InN}$.

\begin{tabular}{ccc}
\hline & $\alpha-\mathrm{GaN}$ & \multicolumn{1}{c}{$\mathrm{InN}$} \\
\hline$E_{0}$ & $3.44 \mathrm{eV}$ & $1.89 \mathrm{eV}$ \\
Conduction band effective mass & & \\
$m_{e}^{z} / m_{0}$ & $0.2^{\mathrm{a}}$ & $0.11^{\mathrm{b}}$ \\
$m_{e}^{t} / m_{0}$ & 0.18 & 0.1 \\
Valence band effective mass parameters & \\
$A_{1}$ & $-6.56^{\mathrm{a}}$ & $-9.28^{\mathrm{b}}$ \\
$A_{2}$ & -0.91 & -0.6 \\
$A_{3}$ & 5.65 & 8.68 \\
$A_{4}$ & -2.83 & -4.34 \\
$A_{5}$ & -3.13 & -4.32 \\
$A_{6}$ & -4.86 & -6.08 \\
Dielectric function & & 8.4 \\
$\epsilon(0)$ & 5.2 & \\
\hline
\end{tabular}

${ }^{\mathrm{a}}$ See Ref. 14.

${ }^{\mathrm{b}}$ See Ref. 18.

tion $x$ is described by $E_{0}\left(\operatorname{In}_{x} \mathrm{Ga}_{1-x} \mathrm{~N}\right)=x E_{0}(\operatorname{InN})+(1$ $-x) E_{0}(\mathrm{GaN})-b x(1-x)$, where $b=1.02 \mathrm{eV}$ is the bowing parameter. ${ }^{14}$ The binary material parameters used in the calculation are listed in Table I. The parameters for $\operatorname{In}_{x} \mathrm{Ga}_{1-x} \mathrm{~N}$ are listed in Table II. Knowing the above parameters, the effective masses can then be determined. ${ }^{15}$ In the $k_{z}$ direction $\left(k_{x}=k_{y}=0\right), \quad m_{\mathrm{HH}}^{\|} / m_{0}=-\left(A_{1}+A_{3}\right)^{-1} ; \quad m_{\mathrm{LH}}^{\|} / m_{0}=-\left(A_{1}\right.$ $\left.+A_{3}\right)^{-1} ; m_{\mathrm{CH}}^{\|} / m_{0}=-A_{1}^{-1}$. In the $k_{x} k_{y}$ plane $\left(k_{z}=0\right)$, $m_{\mathrm{HH}}^{\perp} / m_{0}=-\left(A_{2}+A_{4}-A_{5}\right)^{-1} ; \quad m_{\mathrm{LH}}^{\perp} / m_{0}=-\left(A_{2}+A_{4}\right.$ $\left.+A_{5}\right)^{-1} ; m_{\mathrm{CH}}^{\perp} / m_{0}=-A_{2}^{-1}$ where $\mathrm{HH}, \mathrm{LH}$, and $\mathrm{CH}$ denote heavy hole, light hole, and crystal-field split-off, respectively.

For the continuum state calculations, not only are the model parameter values unknown, but the experimental data for the complex index of refraction of ternary compound $\mathrm{In}_{x} \mathrm{Ga}_{1-x} \mathrm{~N}$ are also not available. Therefore, parameter values for the ternary alloy have been estimated by linear interpolation of the values for $\alpha-\mathrm{GaN}$ and InN. Model parameters for the binary nitrides have been estimated by the acceptance-probability-controlled stimulated annealing (APCSA) algorithm designed for solving multiparameter estimation problems. ${ }^{16}$ The following objective function is used for the model parameter estimation:

TABLE II. Estimated material parameters for $\operatorname{In}_{x} \mathrm{Ga}_{1-x} \mathrm{~N}$.

\begin{tabular}{ll}
\hline \hline & \multicolumn{1}{c}{$\operatorname{In}_{x} \mathrm{Ga}_{1-x} \mathrm{~N}$} \\
\hline$E_{0}$ & $3.45-2.58 x+1.02 x^{2}$ \\
$Q c: Q v$ & $0.67: 0.33$ \\
Conductive band effective mass & $0.2-0.09 x$ \\
$m_{e}^{z} / m_{0}$ & $0.18-0.08 x$ \\
$m_{e}^{t} / m_{0}$ & \\
Valence band effective mass parameters & $-6.56-2.72 x$ \\
$A_{1}$ & $-0.91+0.31 x$ \\
$A_{2}$ & $5.65+3.03 x$ \\
$A_{3}$ & $-2.83-1.51 x$ \\
$A_{4}$ & $-3.13-1.19 x$ \\
$A_{5}$ & $-4.86-1.22 x$ \\
$A_{6}$ & \\
Dielectric function & $5.2+3.2 x$ \\
$\epsilon(0)$ & \\
\hline \hline
\end{tabular}


TABLE III. Estimated model parameters for $\alpha$-GaN and InN.

\begin{tabular}{lcr}
\hline \hline & $\alpha-\mathrm{GaN}$ & $\mathrm{InN}$ \\
\hline$\epsilon_{1 \infty}$ & 0.426 & 1.314 \\
$A_{0}\left(\mathrm{eV}^{1.5}\right)$ & 41.251 & 12.256 \\
$\Gamma_{0}(\mathrm{eV})$ & 0.287 & 0.037 \\
$\alpha_{0}$ & 1.241 & 5.345 \\
$E_{0}^{\prime}(\mathrm{eV})$ & 3.550 & 2.247 \\
$B_{1 A}(\mathrm{eV})$ & 0.778 & 0.361 \\
$B_{1 B}(\mathrm{eV})$ & 0.103 & 1.074 \\
$B_{1 C}(\mathrm{eV})$ & 0.920 & 0.007 \\
$\Gamma_{1 A}(\mathrm{eV})$ & 0.743 & 0.052 \\
$\Gamma_{1 B}(\mathrm{eV})$ & 0.428 & 0.012 \\
$\Gamma_{1 C}(\mathrm{eV})$ & 0.440 & 2.698 \\
$\alpha_{1 A}$ & 0.240 & 5.161 \\
$\alpha_{1 B}$ & 0.011 & 0.574 \\
$\alpha_{1 C}$ & 0.005 & 1.108 \\
$B_{1 A}^{\mathrm{ex}}(\mathrm{eV})$ & 2.042 & 1.243 \\
$B_{1 B}^{\mathrm{ex}}(\mathrm{eV})$ & 1.024 & 0.471 \\
$B_{1 C}^{\mathrm{ex}}(\mathrm{eV})$ & 1.997 & 5.528 \\
$G_{1 A}(\mathrm{eV})$ & 0.0003 & 1.198 \\
$G_{1 B}(\mathrm{eV})$ & 0.356 & 0.521 \\
$G_{1 C}(\mathrm{eV})$ & 1.962 & 4.801 \\
$E_{1 A}(\mathrm{eV})$ & 6.010 & 6.400 \\
$E_{1 B}(\mathrm{eV})$ & 8.182 & 0.001 \\
$E_{1 C}(\mathrm{eV})$ & 8.762 & 0.020 \\
$A_{0}^{\mathrm{ex}}(\mathrm{eV})$ & 0.249 & \\
$G_{0}^{3 \mathrm{D}}(\mathrm{eV})$ & 0.030 & \\
\hline \hline
\end{tabular}

$$
f=\sum_{i=1}^{i=N}\left(\left|\frac{n\left(\omega_{i}\right)-n^{\exp t}\left(\omega_{i}\right)}{n^{\exp }\left(\omega_{i}\right)}\right|+\left|\frac{k\left(\omega_{i}\right)-k^{\exp t}\left(\omega_{i}\right)}{k\left(\omega_{i}\right)}\right|\right)^{2},
$$

where $n\left(\omega_{i}\right)$ and $k\left(\omega_{i}\right)$ are the calculated values, and $n^{\operatorname{expt}}\left(\omega_{i}\right)$ as well as $k^{\operatorname{expt}}\left(\omega_{i}\right)$ are the experimental values of the real and imaginary parts of the index of refraction, respectively, at frequency $\omega_{i}$. The optimal values obtained here for the model parameters are listed in Table III.

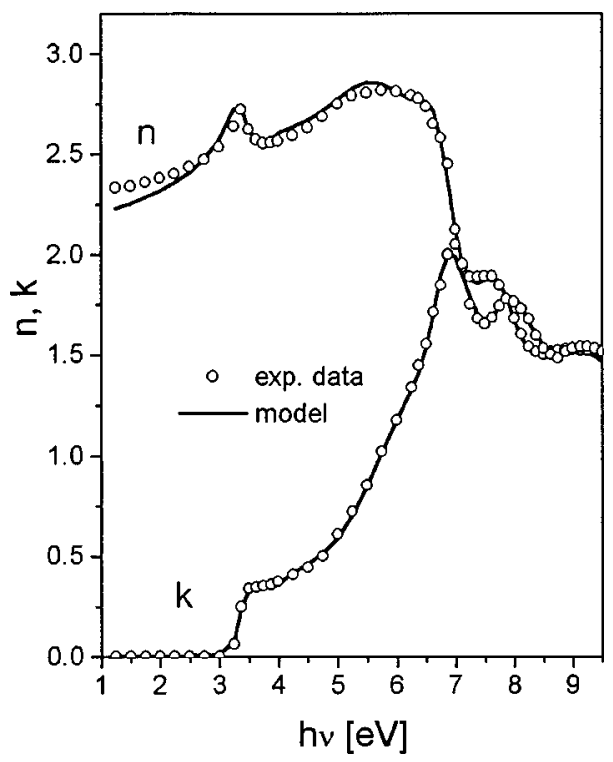

FIG. 1. Comparison between experimental data and calculated result for refractive index and extinction coefficient of $\alpha-\mathrm{GaN}$. The open circles show experimental data from Ref. 2 and the solid line is our calculated result.

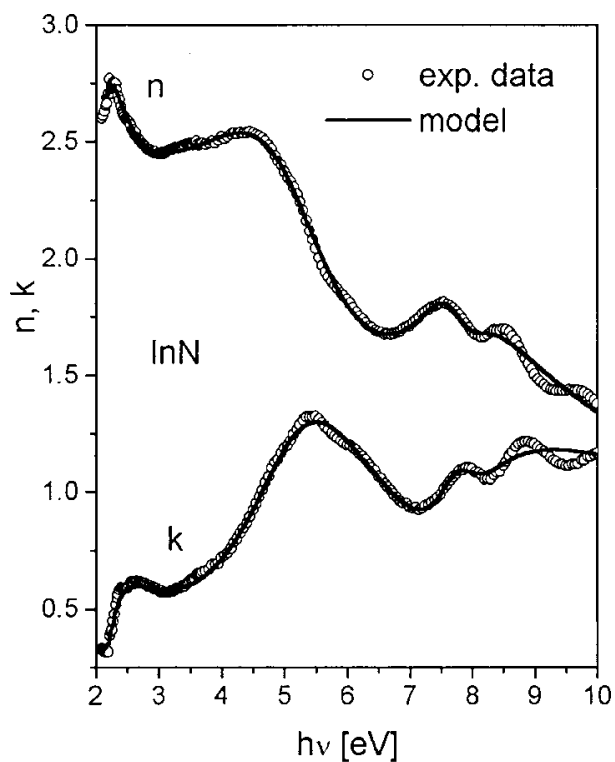

FIG. 2. Comparison between experimental data and calculated result for refractive index and extinction coefficient of $\mathrm{InN}$. The open circles show experimental data from Ref. 14 and the solid line is our calculated result.

Measurements of the optical spectra of $n$ and $k$ for GaN and InN have been reported, ${ }^{2,17}$ and those data have been employed for the model parameter estimation for continuum contributions to the dielectric function. The comparisons between the experimental data and our calculations for $\mathrm{GaN}$ and $\mathrm{InN}$ are presented in Figs. 1 and 2, respectively. Results show that there is good agreement between the experimental data and our calculations.

\section{RESULTS}

The refractive index spectra of the $\mathrm{InGaN} / \mathrm{GaN}$ MQW with the well width equal to 60 and $100 \AA$ and the In content equal to $0.1,0.2$, and 0.3 are now readily determined at room temperature according to the model developed and parameters listed in Tables I-III. The calculated numerical results of exciton binding energy and the bound-state transition energies are listed in Table IV. The theoretical results of the room temperature refractive index for the In content $x$ $=0.1,0.2$ and 0.3 in the energy range from 1 to $9 \mathrm{eV}$ with well width $L_{z}=60 \AA$ and structures with well width $L_{z}=100 \AA$ are presented in Figs. 3 and 4, respectively. In the calculated refractive index spectrum, peaks corresponding to

TABLE IV. Calculated result of exciton binding energy and the bound state transition energy.

\begin{tabular}{lccc}
\hline \hline$X=$ & 0.1 & 0.2 & 0.3 \\
$L_{z}=60 \AA$ & & & \\
\hline Exciton binding energy & $84 \mathrm{meV}$ & $79 \mathrm{meV}$ & $72 \mathrm{meV}$ \\
Bound state transition energy (HH) & $2.9089 \mathrm{eV}$ & $2.3860 \mathrm{eV}$ & $1.8484 \mathrm{eV}$ \\
Bound state transition energy (LH) & $2.9190 \mathrm{eV}$ & $2.3958 \mathrm{eV}$ & $1.8580 \mathrm{eV}$ \\
$L_{z}=100 \AA$ & & & \\
Exciton binding energy & $64 \mathrm{meV}$ & $60 \mathrm{meV}$ & $55 \mathrm{meV}$ \\
Bound state transition energy (HH) & $2.8898 \mathrm{eV}$ & $2.3619 \mathrm{eV}$ & $1.8212 \mathrm{eV}$ \\
Bound state transition energy (LH) & $2.8999 \mathrm{eV}$ & $2.3717 \mathrm{eV}$ & $1.8307 \mathrm{eV}$ \\
\hline \hline
\end{tabular}




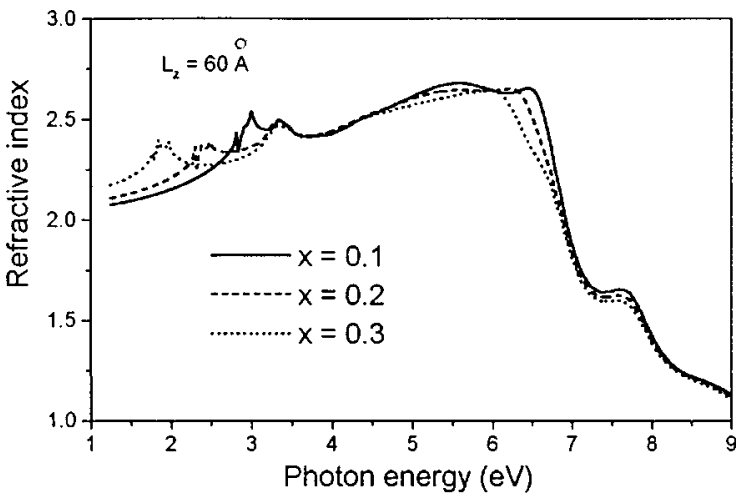

FIG. 3. TE polarization refractive index spectra of $\operatorname{In}_{x} \mathrm{Ga}_{1-x} \mathrm{~N} / \mathrm{GaN}$ MQW with $L_{z}=60 \AA$ for $x=0.1,0.2$, and 0.3 .

contributions from different bound-state transitions can be clearly observed in the energy region between the exciton peak and the barrier band edge. The high amplitude exciton peak is due to the heavy hole exciton. For the QW structure with $L_{z}=60 \AA$ and In content 0.1 (QW barrier band gap of $\approx 3.20 \mathrm{eV}$ ), this peak is located at $2.825 \mathrm{eV}$. It should be followed by a light hole exciton peak at a slightly higher photon energy. Since the two peaks are very close to each other and the light hole peak has a smaller amplitude, they cannot be distinguished from the spectra shown.

The calculated refractive index spectra determined for the In content $x=0.1,0.2$ and 0.3 are plotted in the wavelength range of interest $(0.3-1.0 \mu \mathrm{m})$ in Figs. 5 and 6 for $L_{z}=60 \AA$ and $L_{z}=100 \AA$, respectively. The exciton peak positions of the spectra are located at $0.439 \mu \mathrm{m}(x=0.1)$, $0.537 \mu \mathrm{m}(x=0.2)$ and $0.698 \mu \mathrm{m}(x=0.3)$ for a structure with well width $L_{z}=60 \AA$, and they are at $0.440 \mu \mathrm{m}(x$ $=0.1), \quad 0.539 \mu \mathrm{m}(x=0.2)$ and $0.702 \mu \mathrm{m}(x=0.3)$ for a structure with well width $L_{z}=100 \AA$. Following the exciton peak, the bound-state transition peaks can be observed. With the increase of In content, the band gap of the QW structure decreases. Consequently, the absorption edge shifts to longer wavelengths. It can also be observed that magnitudes of the peaks decrease with increasing In content, while the refractive index value in the transparent region increases.

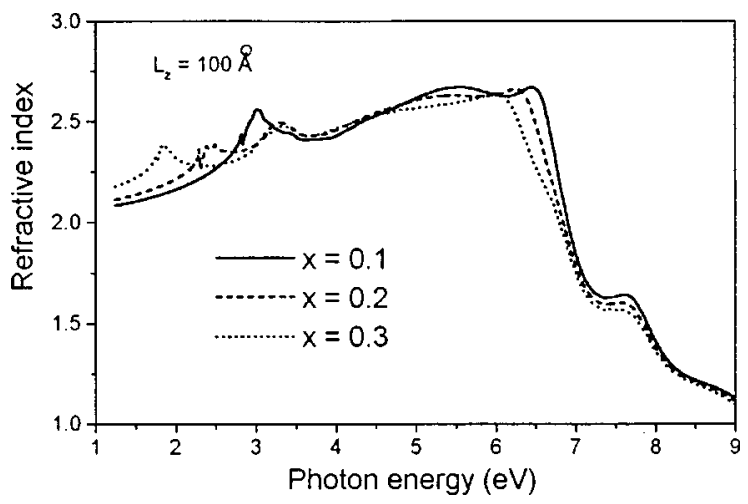

FIG. 4. TE polarization refractive index spectra of $\operatorname{In}_{x} \mathrm{Ga}_{1-x} \mathrm{~N} / \mathrm{GaN}$ MQW with $L_{z}=100 \AA$ for $x=0.1,0.2$, and 0.3 .

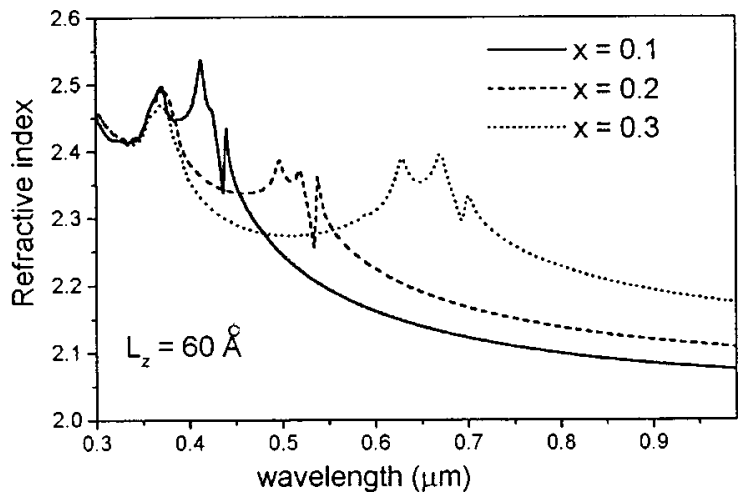

FIG. 5. TE polarization refractive index spectra of $\operatorname{In}_{x} \mathrm{Ga}_{1-x} \mathrm{~N} / \mathrm{GaN}$ MQW with $L_{z}=60 \AA$ for $x=0.1,0.2$ and 0.3 over the spectral range of $0.3-1.0 \mu \mathrm{m}$.

\section{CONCLUSION}

In this work, the dependence of the refractive index of the $\mathrm{In}_{x} \mathrm{Ga}_{1-x} \mathrm{~N} / \mathrm{GaN}$ MQW structure on well width and composition has been studied. The theoretical model presented here is a simple one and yet it is expected to be accurate. It employs semianalytical and semiempirical calculation schemes for the refractive index over a wide spectral range from 1 to $9 \mathrm{eV}$. This model takes into account the continuum state contribution and the QW effect including excitons in the structure. Since the optical properties of the QW system investigated here have- to our knowledge - not been fully studied, there are no reliable experimental data against which our theoretical results can be checked. Our results indicate the expected general trend of the refractive index, and the well-defined peaks obtained in the spectra show the quantum confinement at the band edge of the QW structure. With increasing In content, the absorption edge shifts to lower photon energy and the magnitude of the exciton in the refractive index spectra peaks decreases. The results we have obtained are reasonable and should be able to serve as a reference for the design of photonic devices using this material system. The model presented can also be applied to QW structures of other III-V nitride materials.

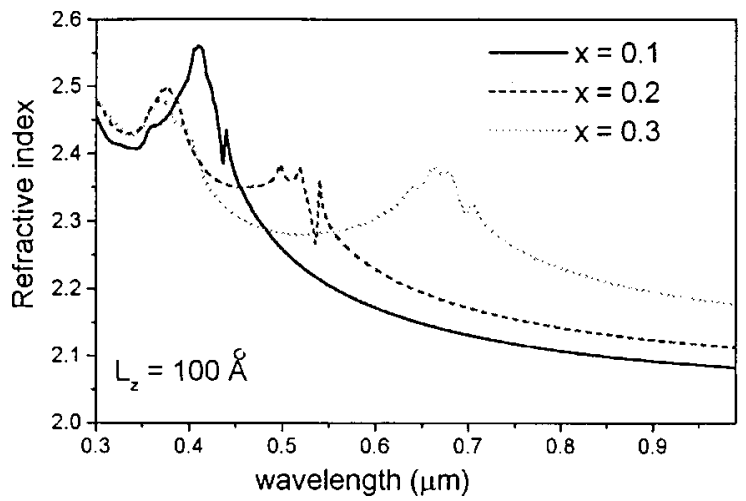

FIG. 6. TE polarization refractive index spectra of $\operatorname{In}_{x} \mathrm{Ga}_{1-x} \mathrm{~N} / \mathrm{GaN}$ MQW with $L_{z}=100 \AA$ for $x=0.1,0.2$ and 0.3 over the spectral range of $0.3-1.0 \mu \mathrm{m}$. 


\section{ACKNOWLEDGMENTS}

The authors would like to thank the RGC earmarked grant of Hong Kong and the University of Hong Kong CRCG research grant for financial support. One of the authors (A.B.D.) was supported by a William Mong Postdoctoral Fellowship of the Faculty of Engineering, the University of Hong Kong.

${ }^{1}$ S. Nakamura and G. Fasol, The Blue Laser Diode: GaN Based Light Emitters and Lasers (Springer, Berlin, 1997).

${ }^{2}$ S. Adachi, Phys. Rev. B 38, 12345 (1988).

${ }^{3}$ S. L. Chuang and C. S. Chang, Phys. Rev. B 54, 2491 (1996).

${ }^{4}$ T. Kawashima, H. Yoshikawa, and S. Adachi, J. Appl. Phys. 82, 3528 (1997).

${ }^{5}$ J. I. Pankove, Optical Process in Semiconductors (Prentice-Hall, Englewood, NJ, 1971), Chap. 4.

${ }^{6}$ G. Bastard, J. A. Brum, and R. Ferreira, "Electronic states in semicon- ductor heterostructures" in Solid State Physics-Advances in Research and Applications, edited by H. Ehrenreich and D. Tumbull (Academic, New York, 1991), Vol. 44, pp. 229-415.

${ }^{7}$ D. J. Ben-Daniel and C. B. Duke, Phys. Rev. 152, 683 (1966).

${ }^{8}$ Y. C. Lee, W. N. Mei, and K. C. Lui, J. Phys. C 15, L469 (1982).

${ }^{9}$ T. F. Jiang, Solid State Commun. 50, 589 (1984).

${ }^{10}$ S. L. Chuang, IEEE J. Quantum Electron. 32, 1791 (1996).

${ }^{11}$ K. S. Chan, J. Phys. C 19, L125 (1986).

${ }^{12}$ A. D. Rakić and M. L. Majewski, J. Appl. Phys. 80, 5509 (1996).

${ }^{13}$ C. C. Kim, J. W. Garland, H. Abad, and P. M. Raccah, Phys. Rev. B 45, 11749 (1992).

${ }^{14}$ A. F. Wright and J. S. Nelson, Appl. Phys. Lett. 66, 3051 (1995).

${ }^{15}$ M. Suzuki, T. Uenoyama, and A. Yanase, Phys. Rev. B 52, 8132 (1995).

${ }^{16}$ A. D. Djurišić, A. D. Rakić, and J. M. Elazar, Phys. Rev. E 55, 4797 (1997).

${ }^{17}$ Q. Guo, O. Kato, M. Fujisawa, and A. Yoshida, Solid State Commun. 83, 721 (1992).

${ }^{18}$ Y. C. Yeo, T. C. Chong, and M. F. Li, J. Appl. Phys. 83, 1429 (1998). 